\title{
The human papillomavirus, cervical cancer and screening strategies: an update.
}

Mahmood Rasool ${ }^{1}$, Sara Zahid ${ }^{2}$, Arif Malik ${ }^{2}$, Irshad Begum², Hani Choudhry ${ }^{3}$, Shakeel Ahmed Ansari $^{1}$, Siew Hua Gan ${ }^{4}$, Mohammad Amjad Kamal ${ }^{5}$, Muhammad Asif ${ }^{6}$, Fawzi Faisal Bokhari ${ }^{7}$, Nawal Helmi $^{3,8}$, Mustafa Zeyadi ${ }^{3}$, Mohammed Hussein Al-Qahtani ${ }^{1}$, Mohammad Sarwar Jamal ${ }^{\text {* }}$

${ }^{1}$ Center of Excellence in Genomic Medicine Research (CEGMR), King Abdulaziz University, Jeddah, Saudi Arabia

${ }^{2}$ Institute of Molecular Biology and Biotechnology (IMBB), University of Lahore, Pakistan

${ }^{3}$ Department of Biochemistry, Cancer Metabolism and Epigenetic Unit, Faculty of Science, Cancer and Mutagenesis Unit, King Fahd Medical Research Center, King Abdulaziz University, Jeddah 21589, Saudi Arabia

${ }^{4}$ Human Genome Centre, School of Medical Sciences, Universiti Sains Malaysia, 16150 Kubang Kerian, Kelantan, Malaysia

${ }^{5}$ King Fahd Medical Research Center, King Abdulaziz University, Jeddah, Saudi Arabia

${ }^{6}$ Department of Biotechnology, BUITEMS, Quetta, Pakistan

${ }^{7}$ Center for Health Studies, Armed Forces Hospitals, Taif Region, Taif, Saudi Arabia

${ }^{8}$ Department of Applied Biochemistry, Faculty of Sciences, University of Jeddah, Jeddah, Saudi Arabia

\begin{abstract}
Cervical cancer, the most common gynaecological malignancy has been strongly correlated with the infection of human papillomavirus (HPV). Different risk factors exaggerate the onset and progression of cervical cancer to malignant and metastatic state. Recent outcomes for the identification of the molecular pathways involved in cervical cancer provide clues about novel bio or oncogenic markers that are proved to be helpful in monitoring the detection of lesion with a high risk of progression in cytological smears and histological specimens. Sexual health programs and public awareness with developing strategies is the ultimate requirement for combating cervical cancer worldwide. The current review discusses mechanism of HPV that links with cervical cancer, various risk factors for the progression of cervical pathologies including smoking, oral contraceptives and sexual factors as well as diagnostic and screening strategies for cervical cancer.
\end{abstract}

Keywords: Papillomavirus, Cervical cancer, Metastasis, Diagnosis, Screening.

Accepted on August 29, 2018

\section{Introduction}

Cervical cancer is accelerated ungoverned growth of anomalous cells of cervix; lower most portion of uterus that opens in vagina [1]. The cancer of cervical epithelium is one of the most widely occurring female malignancy having high death rates in developing countries and most commonly occurring cancer overall the world [2,3]. It has 5 y survival on average, $50 \%$ in developing countries and $66 \%$ in developed countries [4]. A non-communicable disease like cervical cancer creates devastating effects in the developing countries. According to the World Health Organization (WHO) and International Union (IUN) against cancer, 24.6 million people are living with cervical cancer around the world. In 2002 and 2008 , cancer accounted for the deaths of 7.6 million people.
Globally cervical cancer is the fifth and the second most frequent malignancy in men and women respectively. Overall, 715,000 new cervical cancer cases and 542,000 were estimated cervical cancer deaths in Africa. These risks take account of women who does smoke, have history of sexually transmitted diseases, human papillomavirus (HPV) infection, low socio economic position, multiple sexual partner, and suppressed immune system. These factors also support the criteria that cervical cancer is sexually transmitted disease [5]. Nicotine taken by smoking is not itself involved in progression of cervical cancer but it exaggerates it by suppressing immune status [6].

Cervical cancer and its precursor lesion is most important gynaecological revelation of human immunodeficiency virus 
(HIV). Women with HIV are 3 times more risk at developing cervical cancer [7].

HPV infection is very commonly occurring due to its transmission nature, but very few of infections are progressed and meet malignancy and leads toward cervical cancer $[8,9]$. $\mathrm{HPV}$ is main cause for cervical carcinoma but it is proposed that some risks factors are associated with progression of cervical cancer.

The most important and widely contributing risk is high parity and oral contraceptives. If oral contraceptives are used in girls before $17 \mathrm{y}$ at the same time having first sexual intercourse before age of $17 \mathrm{y}$ may increase the risk of cervical cancer more [10]. These two risks are associated with high hormone levels and thus exaggerating the progression of cervical cancer [8].

The circulating levels of estrogen and progesterone are increased due to multi parity and by using long term oral contraceptives [11]. Increased estrogen levels cause the acidification of cervix which ultimately facilitate the squamous cell metaplasia [12].

\section{HPV and Cervical Cancer}

Human papilloma virus is so common in U.S. that almost every sexually active man and woman will transmit the virus during the lifetime. Approximately 10\%-40\% females are infected with HPV at any given time [13]. In spite of its most prevalent nature few people have knowledge of having this HPV infection because their symptoms are seldom noticeable [14].

Human papilloma virus is associated with number of diseases of which most serious is cervical cancer [15]. Almost $20 \%$ of human cancers have infectious agent as cause of occurrence. HPV infection is major etiological cause for the development of cervical cancer [16]. HPV causing cervical cancer has oncogenic HPV types and most commonly occurring HPV-16.

Combined studies have concluded that $99.7 \%$ of all cervical cancers contain HPV and it is highest fraction worldwide for any cause of human cancers [17]. The link between HPV and cervical cancer was first demonstrated in 1980's and identified that 29 types of HPV are involved in causing cancer and confirmed that HPV is present necessarily in all types of cervical cancers $[18,19]$.

Over 150 types of HPV have been known to infect genital tract [20]. Out of these 15 are thought to be known high risk HPV [21]. High risk HPV types are: 16, 18, 31, 33, 35, 39, 45, 51, 52, 56, 58, 59, 68, 73 and 82. HPV 31, 33, 35, 52 and 58 are phylogenetically identical to HPV 16. HPV 39, 45, 59 and 68 are thought to be similar with HPV 18.

Most of the cervical cancer cases are associated with HPV 16 and $1815.2 \%$ and $16.2 \%$ respectively. Other than HPV 16 and 18 other types are also associated with cervical cancer for example 39, 45, 59 and 68. HPV 16 is most frequently found in squamous cell carcinoma (SCC) and HPV 18 is most prevalent in adenocarcinoma (ADC) [22].

\section{Mechanism of Human Papillomavirus-Potential Therapeutic Targets}

Many strains of human papillomavirus have been reported so far which are classified on the basis of their oncogenicity: high risk HPV forms have been involved in the progression of cervical cancers while low risk varieties may rarely be involved in cancerous lesions [23]. There are at present fifteen HPV viruses of high risk category. The two most common HPV varieties are HPV-16 and HPV-18 [18]. The E6 and E7 proteins of high risk strains of HPV are the regulatory proteins involved in oncogenesis. E6 is mainly associated with the lifting of restrictions on cellular proliferation and E7 is involved in cell cycle progression. E2 is also associated with the regulation of cell cycle progression through the expression of E6/E7 which results in the up-regulation of cellular proliferation and enhanced tumerogenesis [18,24]. HPV E2 also possesses an anti-tumor effect when it is introduced into HPV transformed experimental model [25]. High risk E6 and E7 inhibit the innate immune response in immune-competent mice showing its immune-modulatory effect [26]. E6 inhibits the trans-activation function of Interferon regulatory factor-3 (IRF-3) by binding to it whereas E7 represses the transcriptional activity of IRF-1 by binding the IFN- $\beta$ promoter. Since IFN signalling is significantly involved in innate immune response which subsequently activates adaptive immunity, E6 and E7 proteins delay this activation and contribute to the progression of prolonged persistent HPV infection leading to the increased risk of malignant infection [27].

An important and most convincing fact is that E6 protein helps in the association of DNA binding protein expressed in response to DNA damage to the tumor suppressor protein $\mathrm{p} 53$ which causes unscheduled induction of DNA replication resulting in cell cycle arrest or apoptosis [28]. HPV has such a structure that it depends on the cellular DNA synthesis machinery. So it is interpreted that HPV stimulates the progression of S-phase for the replication of its genome. Overexpression of p53 represents a major impairment for viral replication [29]. Binding of E6 with p53 is stimulated by cellular ubiquitin ligase and E6 associated protein (E6-AP) which recruits the ubiquitin complex of enzymes, ubiquitin atinglysines on $\mathrm{p} 53$ causing its proteolysis. Degradation of $\mathrm{p} 53$ arrests the normal growth signals at $\mathrm{G} 1 / \mathrm{S}$ and $\mathrm{G} 2 / \mathrm{M}$ checkpoints in cell cycle which is responsible for the chromosomal instability causing mutational consequences for HPV positive cells [30]. E6 oncoprotein also plays a major role in blocking the degradation of SRC-kinases by E6-AP causing mitotic activity [31].

\section{Risk Factors}

Different risk factors are associated with cervical cancer. These risks are menarche, high parity, an early age at first sexual intercourse, multiple male sex partners, and a history of sexually transmitted diseases, smoking, certain nutritional deficiencies, and low socioeconomic status. There are several independent risk factors for causing cervical cancers including 
age at menarche, age at first delivery, menopause status, and long term estrogen exposure status. The short gap between menarche and start of sexual activity increases the risk for development of cervical cancer [4].

Women having multiple sexual partners significantly increase the risk of developing cervical cancer. And most probably, women reporting approximately 10 partners are at three folds risk of developing cervical cancer. Multiple births and under age at first delivery also exaggerate the risk of developing cervical cancer [32] (Table 1).

Table 1. Classification of HPV types based on risk for carcinogenesis [21].

\begin{tabular}{ll}
\hline Risk classification & HPV types \\
\hline High-risk & $\begin{array}{l}16,18,31,33,35,39,45,51,52,56,58,59,68,73 \text { and } \\
82\end{array}$ \\
\hline Low-risk & $6,11,40,42,43,44,54,61,70,72,81$ and CP6108 \\
\hline
\end{tabular}

\section{Sexual and Reproductive Risk Factors}

Sexual behaviour concludes the exposure to HPV such as multiple sexual partners, unprotected sex, and woman's partner having multiple partner, and early age at sexual intercourse. Out of these risk factors early age at first sexual intercourse (AFSI) is of major importance. Increased risk of HPV is linked with the fact that cervix is immature in adolescents so early sexual intercourse can make it more vulnerable toward the infection of HPV and finally the development of cervical cancer [33]. Early pregnancy is also involved in development of cervical cancer as being major risk factor in the way that women who are married at an early age or get involved in early sexual intercourse may become pregnant, cervical damaged may occur during early age at delivery and ultimately make cervical cells vulnerable to develop cervical cancer [33]. This true with high parity birth or multiple births as trauma to cervix can occur during multiple deliveries. How the early age at first pregnancy and early at sexual intercourse exaggerates the risk of developing cervical cancer may be understood steroid hormonal impact on HPV infection and on immune response of host to HPV during preadolescence and adolescence [33]. Site of cervical cancer development is transformation zone; the susceptibility of this area is linked with its nature. Denudation of stratified epithelium of cervix in between puberty and pregnancy the exposure of cervix to hormonal changes is increased. Increased estrogen levels cause the acidification of cervix which ultimately facilitate the squamous cell metaplasia when the endocervical epithelial avert. This estrogen stimulated meta-plastic changes occurring in the presence of HPV thereby increases risk of cellular transformation that induces neoplasia [12]. It is also believed that these metaplastic changes are increased during trauma and repair process after pregnancies [12]. Increased estrogen can also affect host immune response against HPV. Estrogen levels are three fold higher during follicular phase of the ovarian cycle and pregnancy, these increased levels increases estrogen receptors and their expression in transformation zone [12].

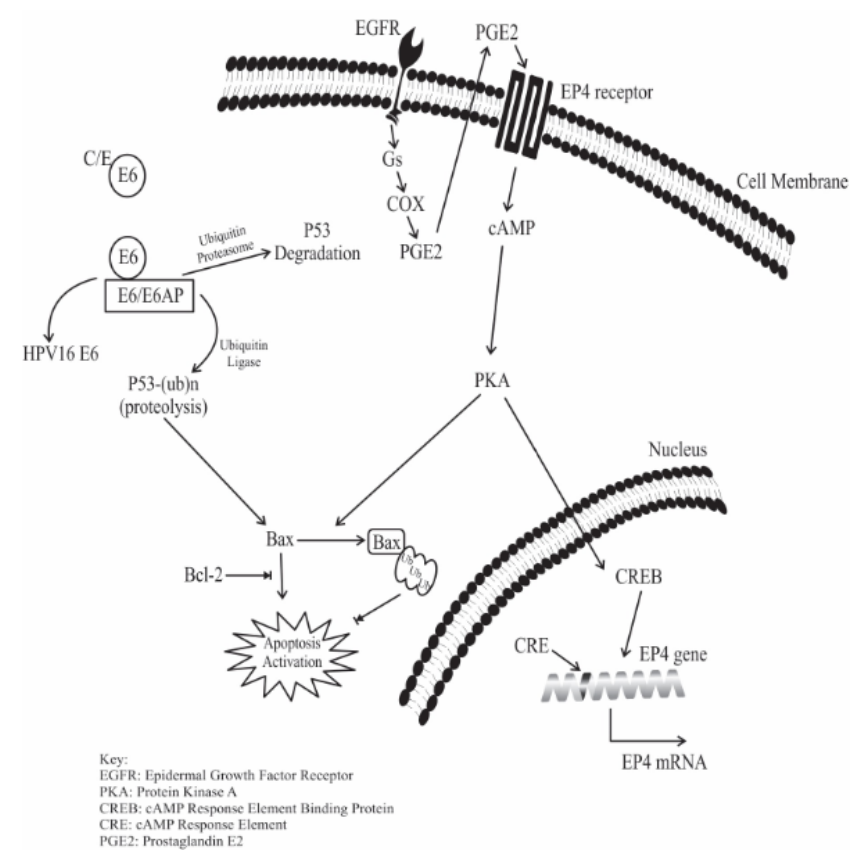

Figure 1. Molecular mechanism of human papilloma virus (HPV) oncogenesis.

\section{Smoking}

Smoking is one of the most important risk factor in the development of cervical cancer. The risk of malignancy is increased with amount and total time period of smoking [10]. The current literature suggests that there is a significant relationship between cervical cancer and smoking, because the women who smoke frequently are at the two fold increased risk of getting cervical cancer [4,10]. Cigarette carcinogens are accumulated in cervical mucus and serve as possible biological factors for the progression cervical cancer. These carcinogens have ultimate transforming effect on the cervical epithelium [4]. Smoking also increases the event of death due to cervical cancer [4]. Researchers believe that these substances damage the DNA of cervix cells and may contribute to the development of cervical cancer. Smoking also makes the immune system less effective in fighting HPV infections. Smoking acts by multiple ways to induce and increase the risk for having cervical cancer (Figure 1). Nicotine rich smoke of cigarettes inhibits apoptosis and subsequently promotes tumor growth [34]. Tobacco increases DNA modification of cervical epithelium through post translational modification and makes favourable circumstances to induce cervical cancer [35]. And secondly nitroso compounds in tobacco induce cervical cancer progression (Figure 2) [35].

\section{Oral Contraceptives and Molecularity}

It is suggested from studies that use of oral contraceptives induces risk of cervical cancer in HPV positive women $[10,35,36]$. Long duration of oral contraceptive use increases the risk of cervical cancer. After the cut down of oral contraceptives the risk associated with is decreased but this hypothesis is proved by limited researches [36,37]. The risk 
associated with oral contraceptives is similar for all types of cervical cancers [38]. The women using combination of estrogen and progesterone currently were at 4 fold increased susceptible towards the risk associated with cervical cancer [39]. There is consistent and high risk associated with long term contraceptive use for developing cervical cancer. It is suggested from past experimental work that progesterone and estrogen present in contraceptives that these two hormones render the host immune response related to HPV evasion [36].

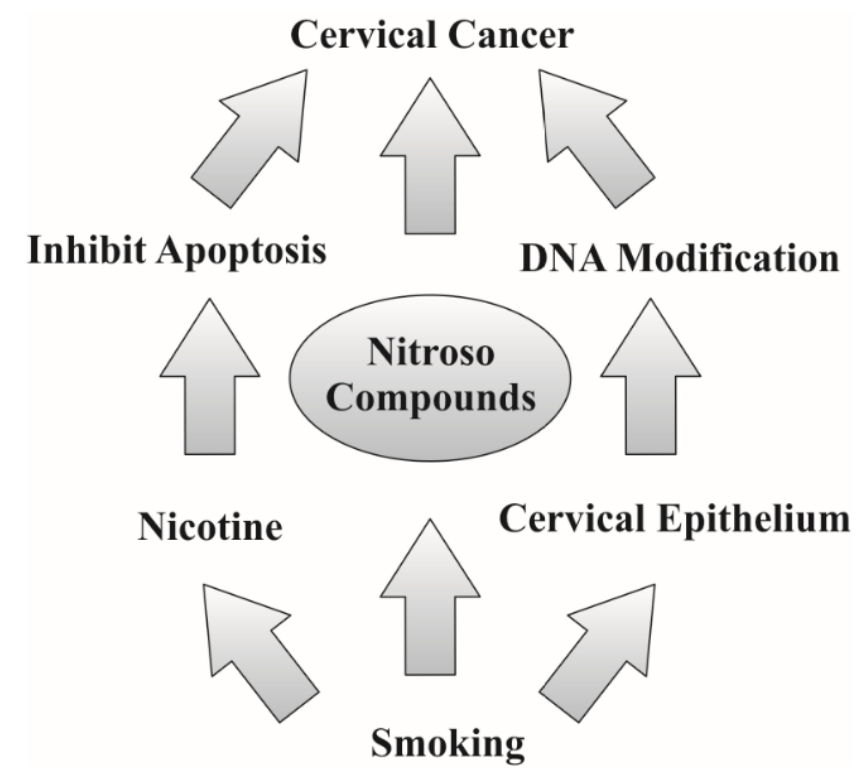

Figure 2. Effect of smoking on cervix.

Contraceptives used at age less than $17 \mathrm{y}$ increases more significantly the risk of having cervical cancer. If oral contraceptives are used in girls before $17 \mathrm{y}$ at the same time having first sexual intercourse before age of $17 \mathrm{y}$ may increase the risk of cervical cancer more [10]. The possible mechanism by which oral contraceptives become potent risk factor indicated that oral contraceptive may increases cervical ectopy which leads to the progression of cervical malignancy [10,37]. Other ways suggested that hormonal recognition elements are present on the transcriptional region of HPV 16 DNA.

Oral contraceptives increase this biding with HPV 16 DNA and thus enhance HPV induced transformation of cervical cells [10]. Oral contraceptives induce local folic acid deficiencies and disturb DNA synthesis, repair and make cell more susceptible towards carcinogens and enhance risk for cervical abnormalities. So long term use of oral contraceptives is the major risk factor for cervical cancer [37].

\section{Education Level and Socioeconomic Status}

Low socioeconomic status (SES) is greatly linked with risk of cervical cancer. The reason behind this link is not fully understood but it is perceived that women having low SES have inadequate screening and showing high parity [40]. Education level serves as a marker for combined risk factor which may occur in women with low SES and become significant risk for cervical cancer. Early events in women's life living low socioeconomic status can modify the potential of HPV inducing cervical cancer. These events may be like early age at sexual intercourse and multiple pregnancies [40].

\section{Hormones as Enhancer}

Another remarkable cofactor linked to the induction of cervical cancer by HPV is estrogen. Oral contraceptives containing estrogen provide good environment for the long term infection of HPV leading to carcinoma of cervical epithelium [39]. Excess and long term use of oral contraceptives is associated with doubling the chance of neoplasia and malignancy induced by HPV [39].

During pregnancy there is increased levels of estrogen which enhance the HPV induced cervical cancer. Some studies also suggested that tumors in cervix expressing both E7 and E6 alone or in concert are estrogen dependent for the onset of progression after initial beginning of infection. Estrogen may acts as carcinogen or as mitogen [41].

\section{Screening and Cytology of HPV}

The majority of the women with cervical cancer experience long asymptomatic period until and unless it is clinically manifested (5). Therefore, regular and proper cytological screening i.e. detecting and removing abnormal tissue or cells in the cervix to treat cervical neoplasia is needed to prevent progression of precancerous lesion to malignant and active cancer. It is proposed that screening work is very low in developing countries because it is not yet confirmed that whether these screening strategies are cost effective in developing countries where cervical cancer is most common cancer next to breast cancer [42]. It is common cancer of low income countries because poor women are less likely to be screened who are more likely to come under exposure of common risks of cervical cancer i.e. smoking and unsafe sex $[42,43]$. Screening is an important step for preventing cervical cancer [44]. The disease burden and worldwide health inequalities would be decreased by improving effective coverage of screening for lowering cervical cancer prevalence [42]. Pap cytology has contributed much in decreasing cervical cancer prevalence; incidence and death rate worldwide [44].

Pap smear testing should begin at age of 18 or after starting sexual life. After having 3 consecutive negative Pap smear results Pap test should be repeated after every 3 y. This way should be adopted in countries having controlled and proper screening system and compiled information regarding that [44]. If this sort of system is missing, pap screening should be repeated after every one year. If mild dysplasia or cervical intraepithelial neoplasia 1 (CIN1) (Table 2) is seen Pap test should be repeated after 6 months. If moderate dysplasia or CIN 2 occurs patient should be advised to perform colposcopy [45]. Despite of successful reduction of cervical incidence by Pap smear some biomarkers are now available for improving cervical cancer screening procedures. These biomarker tools 
are really important for diagnosis and screening of CIN and cervical cancer [46].

Table 2. Three grades for recognizing the cervical intraepithelial neoplasia (CIN).

\begin{tabular}{ll}
\hline CIN 1 & Mild dysplasia \\
\hline CIN 2 & Moderate dysplasia \\
\hline CIN 3 & Severe dysplasia and carcinoma \\
\hline
\end{tabular}

Cervical cancer screening is thought to decrease the risk for spreading of HPV among sexually active persons to about $90 \%$. In the absence of screening there is more chance to develop cancer after the age of 20-25 y. So it is recommended to have Pap screening after every 3 y at least after the age of puberty. Some studies suggested that screening interval should be reduced to $3 \mathrm{y}$ in women having age 25-49 y. For women aged 50 to 64 the interval should be 5 y [47]. Understanding the role of HPV in epidemiology of cervical cancer HPV has gained much importance for the screening of cervical cancer. HPV testing has changed the thinking of women in understanding cervical cancer [48-52]. Pap smear cytology contributed much for controlling cervical cancer. In spite of its successful results Pap is not much perfect. Pap smear test is time consuming and leads to fatigue which causes errors in cytological results. Sampling errors while taking sample from cervix can occur, following laboratory aberrations and thus making it less reliable. Sometimes Pap test shows false positive results which increases patient's anxiety and expenses [44]. All these pitfalls compelled medical technological industry to design new tests with high sensitivity for the detection of precursors of cervical cancer having perfection. HPV testing via viral DNA detection is designed to complete such laps. It detect high risk HPV virus causing cervical cancer [44]. By adding HPV testing to normal cervical cancer screening program the efficacy was increased. HPV testing can be helpful in both ways; as a combining tool with normal Pap smear cytology to raise the efficacy of current Pap cytology program or as a self-contained test for cervical screening. HPV testing doesn't demand much training as with Pap smear test. So it is very good tool to be used as screening in third world countries where overall mortality is high due to cervical cancer $[44,53,54]$.

\section{Conclusion}

Studies have shown that HPV infection is associated with the cervical cancer. Multiple risk factors are responsible for the onset and progression of cervical cancer to malignant and metastatic state. Advancements in molecular targets of this disorder will help for the management of this disorder. Proper and accurate screening will also help for the management of cervical cancer. The most important thing is to give awareness to people for this disease before they get occupied by incurable health issue.

\section{Conflict of Interest}

The authors declare that they have no conflict of financial interests.

\section{References}

1. Kamlesh M. Gynecological malignancies from palliative care perspective. Indian J Palliative Care 2003; 17: 45-51.

2. Atilgan R, Celik A, Boztosun A, Ilter E, Yalta T, Ozercan R. Evaluation of cervical cytological abnormalities in Turkish population. Indian J Ind J Pathol Microbiol 2012; 55: 52-55.

3. Munoz N, Bosch FX, de Sanjose S. Epidemiologic classification of human papillomavirus types associated with cervical cancer. N Engl J Med 2003; 348: 518-527.

4. Odongua N, Chae YM, Kim MR, Yun JE, Jee SH. Association between smoking, screening and death caused by cervical cancer in Korean women. Yonsei Med J 2007; 48: 192-200.

5. Watson RA. Human papillomavirus: confronting the epidemic-an urologist's perspective. Rev Urol 2005; 7: 135-144.

6. Canavan TP, Doshi NR. Cervical cancer. Am Fam Physician 2000; 61: 1369-1376.

7. Ellerbrock TV, Chiasson MA, Bush TJ, Sun XW, Sawo $\mathrm{D}$, Brudney $\mathrm{K}$, Wright TC. Incidence of cervical squamous intraepithelial lesions in HIV-infected women. JAMA 2000; 283: 1031-1037.

8. Burd EM. Human papillomavirus and cervical cancer. Clin Microbiol Rev 2003; 16: 1-17.

9. Arbyn M, Kyrgiou M, Simoens C. Perinatal mortality and other severe adverse pregnancy outcomes associated with treatment of cervical intraepithelial neoplasia: metaanalysis. BMJ 2008; 337: 1284.

10. Gadducci A, Barsoti C, Cosio S, Domenici L, Riccardo Genazzani A. Smoking habits, immune suppression, oral contraceptive use, and hormone replacement therapy use and cervical carcinogenesis. Gynecol Endocrinol 2011; 27: 597-604.

11. Shields TS, Falk RT, Herrero R. A case control study of endogenous hormones and cervical cancer. $\mathrm{Br} \mathrm{J}$ Cancer 2004; 90: 146-152.

12. Louie KS, Sanjose S, Diaz M. Early age at first sexual intercourse and early pregnancy are risk factors for cervical cancer in developing countries. Brit J Cancer 2009; 100: 1191-1197.

13. London S. Acquiring new partner is linked to increased HPV risk among young women. Perspectives on Sexual and Reproductive Health 2003; 35: 150-151.

14. Yim EK, Park JS. The role of HPV E6 and E7 oncoproteins in HPV-associated cervical carcinogenesis. Cancer Res Treat 2005; 37: 319-324.

15. Thomas M, Pim D, Banks L. The role of the E6-p53 interaction in the molecular pathogenesis of HPV. Oncogene 1999; 18: 7690-7700. 
16. Nikitin PA, Luftig MA. The DNA damage response in viral-induced cellular transformation. Br J Cancer 2012; 106: 429-435.

17. Walboomers JM, Jacobs MV, Bosch FX. Human papillomavirus is a necessary cause of invasive cervical cancer worldwide. J Pathol 1999; 189: 12-19.

18. Sur T. The role of HPV 16 E5 protein and its interacting partners in cervical cancer cases. J Tumor 2014; 2: 179-186.

19. Stewart AC, Eriksson AM, Manos MM, Munoz N, Bosch FX, Peto J, Wheeler CM. Intra type variation in 12 human papilloma virus types: A worldwide perspective. J Virol 1996; 70: 3127-3136.

20. Carter JR, Ding Z, Rose B. HPV infection and cervical disease: A review. Aust N Z J Obstet Gynaecol 2011; 51: 103-108.

21. Bosch FX, Qiao YL, Castellsague X. The epidemiology of human papillomavirus infection and its association with cervical cancer. Int J Gynecol Obstetrics 2006; 94: 8-21.

22. Clifford GM, Smith JS, Plummer, Munoz N, Franceschi S. Human papillomavirus types in invasive cervical cancer worldwide: a meta-analysis. Br J Cancer 2003; 88: 63-73.

23. Crum CP, McLachlin CM, Tate JE, Mutter GL. Pathobiology of vulvar squamous neoplasia. Curr Opin Obstet Gynecol 1997; 9: 63-69.

24. Sarian LO, Derchain SF, Yoshida A, Vassallo J, Pignataro F, De Angelo Andrade LA. Expression of cyclooxygenase-2 (COX-2) and Ki67 as related to disease severity and HPV detection in squamous lesions of the cervix. Gynecol Oncol 2006; 102: 537-541.

25. Bermudez-Morales VH, Peralta-Zaragoza O, GuzmánOlea E, García-Carrancá A, Bahena-Román M, AlcocerGonzález JM, Madrid-Marina V. HPV 16 E2 protein induces apoptosis in human and murine HPV 16 transformed epithelial cells and has antitumoral effects in vivo. Tumour Biol 2009; 30: 61-72.

26. Demeret C, Desaintes C, Yaniv M, Thierry F. Different mechanisms contribute to the E2-mediated transcriptional repression of human papillomavirus type 18 viral oncogenes. J Virol 1997; 71: 9343-9349.

27. Peralta-Zaragoza O, Bermudez-Morales V, GutierrezXicotencatl L, Alcocer-Gonzalez J, Recillas-Targa F, Madrid-Marina V. E6 and E7 oncoproteins from human papillomavirus type 16 induce activation of human transforming growth factor betal promoter throughout Sp1 recognition sequence. Viral Immunol 2006; 19: 468-480.

28. Heather LH, Rachel AK, Denise A. Galloway, papillomavirus E6 proteins. Virology 2009; 384: 324-334.

29. Fehrmann F, Laimins LA. Human papillomaviruses: Targeting differentiating epithelial cells for malignant transformation. Oncogene 2003; 22: 5201-5207.
30. Thomas M, Pim D, Banks L. The role of the E6-p53 interaction in the molecular pathogenesis of HPV. Oncogene 1999; 18: 7690-7700.

31. Zur Hausen H. Papillomaviruses causing cancer: Evasion from host-cell control in early events in carcinogenesis. J Natl Cancer Inst 2000; 92: 690-698.

32. Brinton LA, Hamman RF, Huggins, Lehman HF, Levine RS, Mallin K, Fraumeni JF Jr. Sexual and reproductive risk factors for invasive squamous cell cervical cancer. $\mathrm{J}$ Natl Cancer Inst 1987; 79: 23-30.

33. Hong Y, Zhang C, Li X, Lin D, Liu Y. HPV and cervical cancer related knowledge, awareness and testing behaviors in a community sample of female sex workers in China. BMC Public Health 2013; 13: 696.

34. Deng $\mathrm{X}$. Bcl2 family functions as signaling target in nicotine-/NNK-induced survival of human lung cancer cells. Scientifica 2014; 2014: 1-7.

35. Janet RD, Margaret M, Madeleine BM. The relationship of human papillomavirus-related cervical tumors to cigarette smoking, oral contraceptive use, and prior Herpes simplex virus type 2 infection. Cancer Epidemiol Biomarkers Prev 1996; 8: 541-548.

36. Ylitalo N, Sorensen P, Josefsson A. Smoking and oral contraceptives as risk factors for cervical carcinoma in situ. Int J Cancer 1999; 81: 357-365.

37. Kjellberg L, Hallmans G, Ahren AM. Smoking, diet, pregnancy and oral contraceptive use as risk factors for cervical intra-epithelial neoplasia in relation to human papillomavirus infection. $\mathrm{Br} \mathrm{J}$ Cancer 2000; 82: 1332-1338.

38. Wells SI, Aronow BJ, Wise TM, Williams SS, Couget JA, Howley PM. Transcriptome signature of irreversible senescence in human papillomavirus-positive cervical cancer cells. Proc Natl Acad Sci USA 20003; 100: 7093-7098.

39. Jeffery MA, Peter MH, Douglas H. Chronic estrogen induced cervical and vaginal squamous carcinogenesis in human papillomavirus type 16 transgenic type mice. Proc Natl Acad Sci 1996; 93: 2930-2935.

40. Louie KS, de Sanjose S, Diaz M. Early stage at first sexual intercourse and early pregnancy are risk factors for cervical cancer in developing countries. Br J Cancer 2009; 100: 1191-1197.

41. Tiffany B, Paule FL. Estrogen contributes to the onset, persistence, and malignant progression of cervical cancer in a human papillomavirus-transgenic mouse model. Proc Natl Acad Sci 2005; 102: 2490-2495.

42. Emmanuel G, Stella N, Ziad O. Coverage of cervical cancer screening in 57 countries: low average levels and large inequalities. Pmed 2008; 5: 863-868.

43. Parveen S, Sajjad R, Masood M, Usmani HA, Sadiq R, Yunus N, Asghar S. Cervical cancer: Outcome of treatment and cause of failure. J Pakistan Med Association 2006; 56: 436- 440.

44. Ratnam S, Franco EL, Ferenczy A. Human papillomavirus testing for primary screening of 
cervical cancer precursors. Cancer Epidemiol Biomarkers Prevention 2000; 9: 945-951.

45. Eduardo LF, Eliane DF, Alex F. Cervical cancer: epidemiology, prevention and the role of human papillomavirus infection. Canadian Med Association J 2001; 164: 1017-1025.

46. Lobato S, Tafuri A, Fernandes PA, Caliari MV, Silva MX, Xavier MA, Vago AR. Minichromosome maintenance 7 protein is a reliable biological marker for human cervical progressive disease. J Gynecol Oncol 2012; 23: 11-15.

47. Canfell K, Barnabas R, Patnick J, Berak V. The predicted effect of changes in cervical screening practice in the UK: results from a modeling study. Brit J Cancer 2004; 91: 530-536.

48. Waller J, McCaffery K, Nazroo J, Wardle J. Making sense of information about HPV in cervical screening: a qualitative study. Brit J Cancer 2005; 92: 265-270.

49. Hacer GS, Lale T, Wright TC Jr. Determining nursemidwives' knowledge of the PapSmear test and their rate of being tested in Turkey. Asian Pac J Cancer Preven 2011; 2: 17.

50. Gopal K. Singh disclosures community health, rural-urban trends and patterns in cervical cancer mortality, incidence, stage, and survival in the United States. 2012; 37: 217-223.
51. WHO/ICO. Information centre on HPV and cervical cancer (HPV Information Centre), author Summary report on HPV and cervical cancer statistics in Ethiopia. World Health Organisation, Ethiopia 2014.

52. Echelman D, Feldman S. Management of cervical precancers: Global perspectives. Hematol Oncol Clin N Am 2012; 26: 32-44.

53. Yesuf T. Survival and associated factors among cervical cancer patients in Black Lion Hospital, Addis Ababa, Ethiopia. JHIA 2012.

54. Getahun F, Mazengia F, Abuhay M, Birhanu Z. Comprehensive knowledge about cervical cancer is low among women in Northwest Ethiopia. BMC Cancer 2013; 14: 2 .

\section{*Correspondence to}

Mohammad Sarwar Jamal

King Fahd Medical Research Center

King Abdulaziz University

Jeddah

Saudi Arabia 